\title{
USO TERAPEUTICO DEL APRENDIZAJE DE UN IDIOMA
}

\author{
THE THERAPEUTIC USE OF LANGUAGE LEARNING
}

Cristina de Diego Alonso. cristinadediegoalonso@gmail.com

Patricia Jovellar Isiegas. jovejita@hotmail.com

Terapeutas ocupacionales

\section{Resumen}

A través de los ojos de dos estudiantes de Terapia Ocupacional durante una beca Erasmus llevada a cabo en un psiquiátrico en Bélgica, se realiza un análisis de la actividad: "aprender un idioma" como parte de la terapia de pacientes en el área de la salud mental.

Se ponen de manifiesto todos los aspectos que se pueden trabajar (habilidades cognitivas, relaciones interpersonales y sociabilidad, comunicación, autoestima, función ocupacional y la incidencia de las alucinaciones auditivas), y cómo todos ellos surgen de manera natural cuando se lleva a cabo el aprendizaje de un idioma. Veremos cómo una actividad propositiva y significativa para el paciente se convierte en una actividad terapéutica

\begin{abstract}
Abstrac
From two Occupational Therapy's students point of view during an Erasmus in a psychiatric in Belgium, the article explain an an anlysis of: "language learning” as a part into a therapy with patients in mental health.

The article tries to show all aspects we can develop in patients (cognitive skills, interpersonal relations and socialability, self steem, occupational fuctioning and how to mitigate auditive hallucinations), and how all of them come up in a natural way during a language learning. In conclusion how a meaningful activity to the patient became in a therapeutic activity.
\end{abstract}

\section{Palabras clave}

Salud mental, aprender un idioma, actividad propositiva, relacción terapéutica , función ocupacional.

\section{Key words}

Mental health, language learning, activity with meaning, therapeutics relationship, occupational fuctioning.

\section{¿Qué hacemos allí?}

Durante nuestro $2^{\circ}$ año de estudiantes de Terapia Ocupacional en la Escuela Universitaria de Ciencias de la salud de Zaragoza decidimos solicitar una beca Erasmus. En ese momento todavía no éramos conscientes de lo que nos sucedería después. El motivo fundamental de esta petición fue el tener la oportunidad de ver los diferentes métodos de trabajo desde la Terapia Ocupacional en otros países, en los que además nuestra profesión está más asentada e introducida en el mundo sanitario, con la finalidad de tener una perspectiva más amplia y de practicar un segundo idioma como es el inglés.

Después de unos intensos meses llenos de preparativos e incertidumbres todo estuvo listo .Nos embarcamos en lo que al final resultó ser una gran aventura con destino Bélgica, que duraría tres inolvidables meses. Las primeras semanas fueron un poco caóticas: nuevo ambiente, otro idioma, distinta cultura, hábitos, costumbres...en definitiva, todo un mundo nuevo y diferente que se abría ante nosotras ofreciéndonos una multitud de nuevas experiencias. Nosotras, como futuras 
Terapeutas Ocupacionales supimos adaptarnos al entorno, que enseguida comenzó a parecernos muy familiar y que todavía ahora recordamos de forma muy positiva.

\section{¿Dónde?}

Uno de los centros dónde realizamos las prácticas clínicas fue el Psiquiátrico Universitair Centrum St. Jozef (Kortenberg,) donde tuvimos la oportunidad de formar parte de un novedoso proyecto: clases de español como actividad terapéutica desde T.O. para enfermos psiquiátricos crónicos, motivo por el cual decidimos hacer este artículo.

En Saint Jozef hay 4 salas que están enfocadas al tratamiento de pacientes con psicosis. Una de estas cuatro salas St. Agnes está exclusivamente enfocada a la admisión de pacientes psicóticos en crisis serias. La mayoria de las veces, después de la intervención en la crisis, los pacientes son trasladados a una sala terapéutica donde además de medicación se utilizan otros métodos para el tratamiento. En la sala St. Joris los pacientes nunca han tenido antes un tratamiento residencial. El promedio de edad es aproximadamente 20 años. A los pacientes se les ofrece un programa que incluye diferentes fases y después de un periodo de tres a seis meses, se les da permiso para irse y se les proporciona un cuidado posterior. La mayoría de ellos tienen un buen pronóstico. En la sala St Mónica, encontramos a aquellos pacientes que son admitidos para bastante tiempo y que ya han tenido varias admisiones. La rehabilitación, que en general comienza por encontrar un lugar para vivir y algo que hacer, es la meta en esta sala ${ }^{1}$.

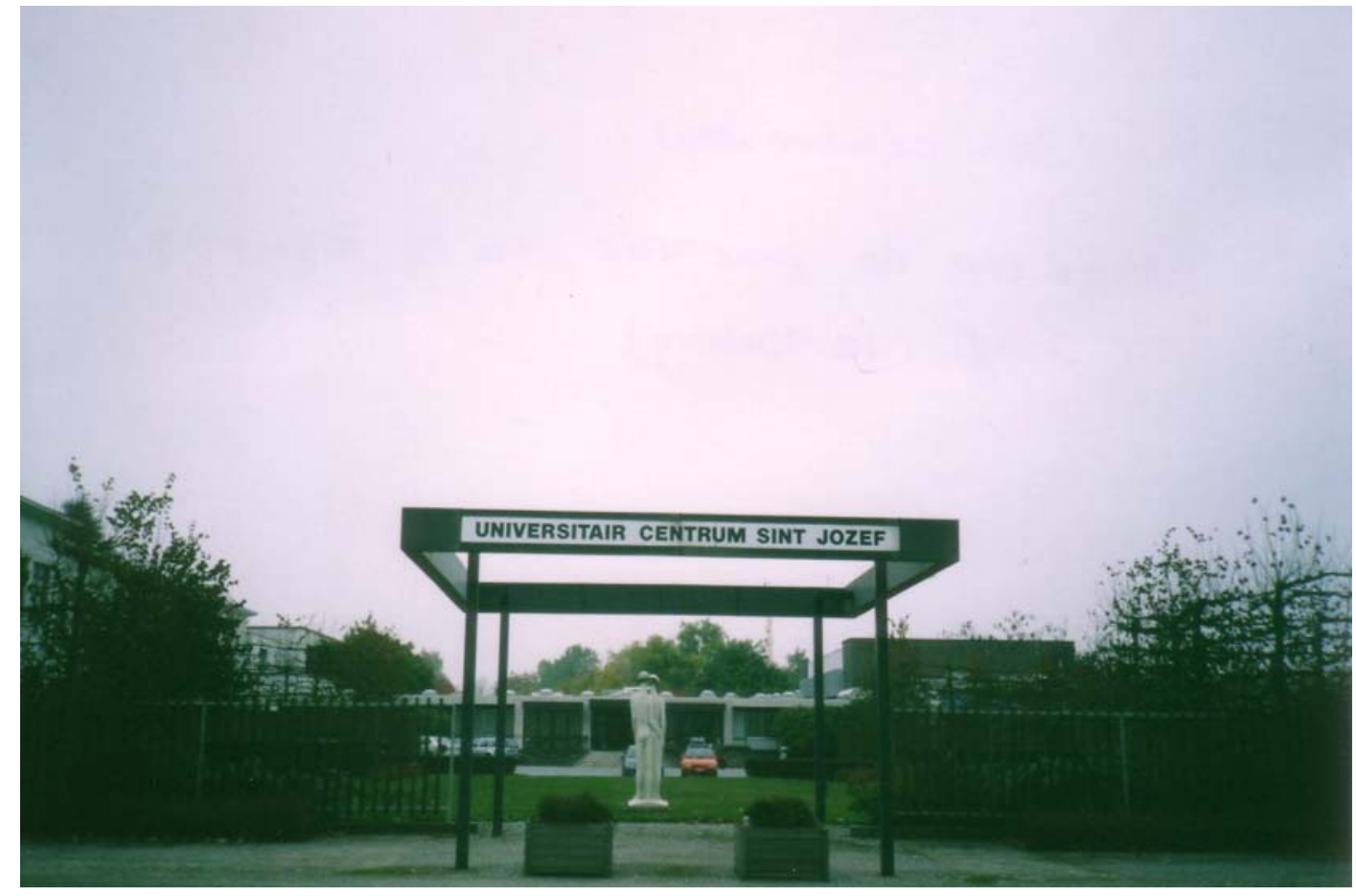

Ilustración 1 (Univeritair Centrum St-Jozef)

En St Franciscus, unidad en dónde nos encontrábamos, cuidamos a aquellos pacientes resistentes a la terapia que no pueden manejar la presión, incluso por pequeña que esta sea, en las salas anteriormente mencionadas. Como término medio, ellos no tienen la perspectiva de ser parte de una vida social otra $v^{2} z^{2}$. Se trata de pacientes crónicos que cuentan con una larga historia de enfermedad en su pasado, lo que ha generado en su presente una serie de handicaps que le impiden el desarrollo de una vida normal y con los cuales tienen que aprender a vivir el resto de su vida.

\footnotetext{
${ }^{1}$ Gary Kielhofner. Model of Human Occupation: Theory and application 3rd edition, Lippincott Williams\&Wilkins, 2002. Capítulo 22: Appling MoHO to clients who are cognitively impaired, Luc Vercruysse, p.398-401.

${ }^{2}$ Gary Kielhofner. Model of Human Occupation: Theory and application 3rd edition, Lippincott Williams\&Wilkins, 2002. Capítulo 22: Appling MoHO to clients who are cognitively impaired, Luc Vercruysse, p.398-401.
} 
Debido a su enfermedad estos pacientes son muy vulnerables ante situaciones desconocidas o en las que se sienten presionados, manifestándose en altos niveles de estrés y ansiedad que pueden desencadenar un nuevo episodio psicótico. Además hay que tener en cuenta que, a pesar de que normalmente se encuentran en una situación estable gracias al tratamiento farmacológico, se siguen manifestando en ellos determinados síntomas, como las alucinaciones e ilusiones, con las que tendrán que aprender a convivir de por vida.

Siendo la persona el centro del enfoque terapéutico y teniendo en cuenta su patología se decide el tratamiento más (adecuado, indicando la posibilidad de seguir distintas terapias: Terapia Ocupacional, Músico-terapia, Arte-terapia, Terapia psicomotriz, Terapia psicológica...coordinadas entre sí en busca siempre de los mismos objetivos.

\section{¿Con quién?}

Dentro del amplio abanico de trastornos psicóticos nos centraremos en explicar brevemente los que con mayor frecuencia se encontraban en la unidad de St Franciscus:

Esquizofrenia: es una alteración que persiste durante por lo menos 6 meses e incluye por lo menos 1 mes de síntomas de la fase activa (ideas delirantes, alucinaciones, lenguaje desorganizado, comportamiento catatónico o gravemente desorganizado) y síntomas negativos (aplanamiento afectivo, alogia o abulia). Se puede manifestar como esquizofrenia paranoide, desorganizada, catatónica, indiferenciada o residual ${ }^{3}$.

Los síntomas característicos pueden conceptualizarse como pertenecientes a dos amplias categorías: positivos y negativos. Los síntomas positivos parecen reflejar un exceso o distorsión de las funciones normales, mientras que los negativos parecen reflejar una disminución o pérdida de las funciones normales. Los síntomas positivos incluyen distorsiones o exageraciones del pensamiento inferencial (ideas delirantes), la percepción (alucinaciones), el lenguaje y la comunicación (lenguaje desorganizado), y la organización comportamental (comportamiento gravemente desorganizado o catatónico). Los síntomas negativos comprenden restricciones del ámbito y la intensidad de la expresión emocional (aplanamiento afectivo), de la fluidez y la productividad del pensamiento y el lenguaje (alogia), y del inicio del comportamiento dirigido a un objetivo (abulia).

El diagnóstico implica el reconocimiento de una constelación de signos y síntomas asociados a un deterioro de la actividad social o laboral.

- Trastorno esquizoafectivo: es una alteración en la que se presentan simultáneamente un episodio afectivo y los síntomas de la fase activa de la esquizofrenia, y está precedida o seguida por al menos 2 semanas de ideas delirantes o alucinaciones sin síntomas importantes de alteración del estado de ánimo ${ }^{4}$.

- $\quad$ Trastorno delirante: se caracteriza por al menos un mes de ideas delirantes no extrañas sin otros síntomas de la fase activa de la esquizofrenia.

\section{Filosofía de trabajo}

Así pues la filosofía con la que se trabaja en St. Francisco debe adecuarse a estas características. Por ello la unidad queda abierta a los pacientes durante todo el día y ofrece multitud de actividades ocupacionales, siendo la persona la que decide CÓMO, CUÁNDO Y QUÉ quiere realizar en Terapia Ocupacional. Los cuatro pilares básicos de nuestra rehabilitación nos acercan a que es necesaria la medicación, estructura del día fijada, una persona designada en la que él pueda confiar y con la que pueda contar (enfermera individual) y el apoyo de la familia ${ }^{5}$.

El rol del terapeuta es guiar y motivar al paciente en estas decisiones, negociando con él y proporcionándole un ambiente con niveles de estrés y ansiedad que le resulten soportables a la vez que estimuladores. No podemos hablar de un tratamiento en el sentido estricto de la palabra, es decir, prescrito por el terapeuta, puesto que es el paciente el que elige su propio tratamiento. Será

\footnotetext{
${ }^{3}$ DSM-IV: Manual diagnóstico y estadístico de los trastornos mentales 4a ed. Barcelona, Masson S.A, 1995. PG 279-280

Citar al final del trabajo

${ }^{4}$ DSM-IV: Manual diagnostico y estadístico de los trastornos mentales $4^{\mathrm{a}}$ ed. Barcelona Masson S.A, 1995. PG 279-280

${ }^{5}$ Gary Kielhofner. Model of Human Occupation: Theory and application 3rd edition, Lippincott Williams\&Wilkins, 2002. Capítulo

22: Appling MoHO to clients who are cognitively impaired, Luc Vercruysse, p.398-401.
} 
siempre bajo supervisión del terapeuta, tratando de lograr la máxima independencia y autonomía posibles, aprendiendo a convivir con los síntomas residuales de su enfermedad. Este será el objetivo principal planteado inicialmente dentro de la clínica, que en un futuro y si es posible, será llevado a cabo en sociedad.

Basándonos en las características anteriormente expuestas del paciente crónico y siguiendo estas pautas de actuación, fue como desarrollamos las clases de español. Luc Vercruysse, Terapeuta Ocupacional de St Jozef, sabía que varios pacientes estaban interesados en aprender español u otros idiomas tras utilizar diversas escalas como el "Interest Checklist” y “OPHI-II" pertenecientes al Modelo de Ocupación Humana .Así que con nuestra llegada se valoró la posibilidad de realizar un curso de español: “¿Qué mejor actividad terapéutica que la enseñanza de nuestro propio idioma?”

Decidimos fijar 2 días a la semana para dar las clases. Uno por la mañana y otro por la tarde en dónde se repetiría la misma lección, siendo flexibles con aquellos pacientes que debido a los efectos secundarios de los fármacos u otras causas no podían acudir por la mañana. Con la misma finalidad se les daba la opción de un descanso en la mitad de los 60 minutos que duraba la clase. También podían abandonarla si por motivos de estrés, ansiedad u otros, no se encontraban bien, siendo posible volver cuando estuvieran animados y dispuestos a seguir aprendiendo. Una de las cosas fundamentales en la vida de estas personas es proporcionarles orden y estructura. Un entorno estructurado favorece un ambiente relajado en el que la persona se maneja sin experimentar estrés y ansiedad, por ello las partes de las que constaban las clases siempre eran las mismas y seguían un mismo orden:

1- Buenos días

2- Repaso de la lección anterior

3- Nuevos conceptos

4- Conversación y despedida

Ilustración 2 (Ejemplo de una clase)

La hoja correspondiente a cada clase se les daba con anterioridad, ofreciéndoles la oportunidad de conocer de ante mano cómo se iba a desarrollar cada sesión.

\section{Objetivos y Metodología: ¿Por qué y cómo?}

Con el desarrollo de las clases de español como actividad terapéutica nos propusimos conseguir una serie de objetivos generales y otros más específicos:

1- Potenciar las habilidades cognitivas

2- Fomentar las relaciones interpersonales y sociabilidad (comunicación)

3- Mejorar el autoconcepto y la autoestima

4- Potenciar la función ocupacional

5- Aminorar la incidencia de las alucinaciones auditivas

A continuación los expondremos justificando en primer lugar el porqué de su elección y en segundo lugar cómo se llevaron a cabo:

\section{Potenciar las habilidades cognitivas:}

Seguiremos el marco de referencia cognitivo. Este marco esta basado en el procesamiento de la información y tiene como referente las llamadas facultades mentales básicas como la sensopercepción, la atención, la memoria, el lenguaje...Los mecanismos de procesamiento de estos elementos se basan en la seriación, en la clasificación , en la inclusión-exclusión y pensamiento lógico. Y como actividades ocupacionales más representativas tenemos el juego representativo en la infancia y la resolución de problemas en la edad adulta. El marco de referencia cognitivo está en la 
base del aprendizaje relacionado con el manejo simbólico y la automatización de los hábitos sociales ${ }^{6}$.

Estimular la atención, la concentración y la memoria

El deterioro cognitivo aparece como un síntoma frecuente. Esto repercute profundamente en las actividades diarias del paciente, motivo por el cuál el desarrollo de aspectos como atenciónconcentración-memoria fueron pilares básicos en la realización de las clases.

La realización de tareas con componentes, en cuanto a habilidades y destrezas, cognitivos importantes se estructuran según el siguiente orden de complejidad, de menor a mayor, para mejorar la: atención, percepción, memoria a corto plazo, memoria a largo plazo, memoria de datos y hechos y memoria de relaciones entre datos ${ }^{7}$.

Al comienzo de cada sesión, un objetivo importante era que la persona fuera capaz de mantener el suficiente nivel de atención-concentración como para seguir toda la clase. Para ello tratamos de preparar las sesiones de forma dinámica, participativa y atractiva para los pacientes. Esto junto a la estructuración proporcionada a través del guión escrito al comienzo de la actividad, hacía que fueran capaces de seguirlas sin problemas, pudiendo abandonarlas cuando sus niveles de frustración aumentaran.

La memoria era potenciada tanto a corto como a largo plazo. Como puede verse en la estructura de las clases, uno de los apartados está dirigido a recordar conceptos aprendidos anteriormente (memoria a largo plazo).Mientras que otros eran reservados al aprendizaje de nuevos conceptos, apoyados por ejercicios prácticos que favorecían su asimilación (memoria a corto plazo).

Incrementar la fluidez en el lenguaje y como consecuencia en la comunicación

En estos pacientes se observa una pobreza del lenguaje, tanto en su expresión como en su contenido, y también una tangencialidad de sus respuestas. Todo ello se puede ver reflejado en cierto grado de incomunicación. Esto se ve agravado también por la falta de sentido de la realidad, que se manifiesta en alteraciones de los aspectos temporo-espaciales y de conciencia corporal relacionados con las actividades de la vida diaria (AVDs) ${ }^{8}$.

En relación con las destrezas para la comunicación y la interacción (ACIS) tenemos que la comunicación en el dominio físico (gestual, corporal) adolece de una falta de expresividad. En determinadas formas clínicas llega a estar totalmente anulada. En general el intercambio de información es fluctuante. Las relaciones fracasan en el ámbito de la colaboración, en el cumplimento de lo pactado, no se respeta el territorio y las competencias del otro, resultando el rapport difícil y en ocasiones imposible ${ }^{9}$.

La enseñanza del vocabulario siempre hacía referencia a objetos concretos y habituales en su vida, evitando el uso de palabras abstractas que les desligan de la realidad. Por ello los temas que nosotras seleccionamos para las clases fueron: los colores, los números, las horas, los días de la semana, las comidas y bebidas, las partes del cuerpo, algunos verbos y frases útiles, sin olvidar aspectos de la cultura española como gastronomía y geografía. En las clases potenciábamos el aprendizaje de nuevas palabras, su significado y su verbalización, resaltando las propiedades y funciones de los objetos enseñados (gnosis). De esta manera enriquecíamos la pobreza de su lenguaje y favorecíamos la intercomunicación

Mejorar la Inhibición-expectancia

\footnotetext{
${ }^{6}$ Jesús Gómez Tolón y Roberto Salvanés Pérez. Terapia ocupacional en psiquiatría. Zaragoza, Mira editores, 2003. Pág. 21-22

${ }^{7}$ Jesús Gómez Tolón .Fundamentos metodológicos de la terapia ocupacional. Zaragoza, Mira editores, 1997 Pág. 109- 110

89 Jesús Gómez Tolón y Roberto Salvanés Pérez. Terapia ocupacional en psiquiatría. Zaragoza, Mira editores, 2003. Pág. 45 y 55
} 
Las alteraciones en la inhibición y expectancia se dan frecuentemente en estas personas, es decir, la perseverancia y distraibilidad, que pueden quedar reflejados en una dificultad de control por el lenguaje interno de la conducta ${ }^{10}$.

En las sesiones potenciábamos estos aspectos planteando actividades como por ejemplo exponer una pregunta a todo el grupo de manera que la respuesta debía ser individual. Tenían que respetar su turno y tener en cuenta las respuestas anteriores de sus compañeros.

Gracias a la función mediadora del lenguaje se produce una inhibición progresiva de las reacciones estímulo-respuesta (frecuentes en estas personas). La mediación del lenguaje se inicia mediante la obediencia a las consignas verbales del adulto. El seguimiento de las normas del juego también inhibe esta tendencia a las reacciones estímulo-respuesta. La inhibición y la atención expectante son el resultado de esta función mediadora. La inhibición neutraliza los estímulos del medio (control espacial) que pueden interferir con las órdenes verbales internas o externas, y la atención expectante crea un estado mental de espera (control temporal) de un acontecimiento previamente anunciado $^{11}$.

Estos aspectos fueron trabajados a lo largo de todas las sesiones, pero cuando realmente pudimos ver el resultado fue en el "Remember's Game" que realizamos el último día de curso. En él demostraron tener la capacidad de obedecer nuestras órdenes verbales y de seguir las normas del juego, dando paso a un comportamiento progresivamente dirigido por consignas sociales.

Entrenar la orientación a la realidad (aspectos temporo-espaciales y corporales)

El sentido de la realidad se intentará restaurar o mejorar mediante el incremento de la conciencia corporal y la conciencia de los aspectos temporales y espaciales relacionados con las actividades de la vida diaria. El adiestramiento en actividades que involucren de manera predominante la conciencia corporal será el primer peldaño para crear o restaurar un cierto sentido de la realidad y la base de la diferenciación entre el “yo" y los “otros”12.

En nuestras sesiones aprovechamos el tema del cuerpo para, siguiendo la pauta de PiagetHead, trabajar el dibujo de la figura humana y la denominación de las distintas partes del cuerpo, primero sólo del propio individuo y después del cuerpo del terapeuta o de un muñeco. Siendo un procedimiento de toma de conciencia corporal con progresiva descentración (refuerzo de la diferenciación entre “yo" y los “otros”)

En referencia a los aspectos temporales, empezamos por resaltar el contraste entre el día laborable de los domingos con actividades claramente diferenciadas. Después diferenciamos entre la mañana y la tarde utilizando las actividades de la vida diaria en relación con la alimentación, el vestido, la higiene o el descanso. Para la diferenciación de las estaciones utilizamos las acciones y ropas típicas de cada una de ellas. Y tan pronto como fue posible introdujimos las referencias al calendario, a los días de la semana y a las horas del reloj.

Otra herramienta que utilizamos para fomentar el contacto con la realidad fue el "Buenos Días” del comienzo de cada clase en el que se establecía una pequeña conversación por parejas y se preguntaban cosas como: ¿Qué día es hoy? ¿En qué mes y año nos encontramos? ¿Cómo te llamas? ¿De dónde eres? ¿Qué hiciste ayer?

Queremos destacar que todos estos aspectos surgen de forma natural, ya que constituyen un conjunto de gestos normalizados en lo que supone aprender un idioma. Así pues, lo único que tuvimos que hacer fue utilizarlos de manera terapéutica mediante el planteamiento previo de unos objetivos.

\footnotetext{
10_ ${ }^{12}$ Jesús Gómez Tolón y Roberto Salvanés Pérez. Terapia ocupacional en psiquiatría. Zaragoza, Mira editores, 2003. Pág 45, Pág 64-65

${ }^{11}$ Jesús Gómez Tolón. Habilidades y destrezas en terapia ocupacional.. 1º ed. Zaragoza, Mira editores, 2000. Pág 120.
} 

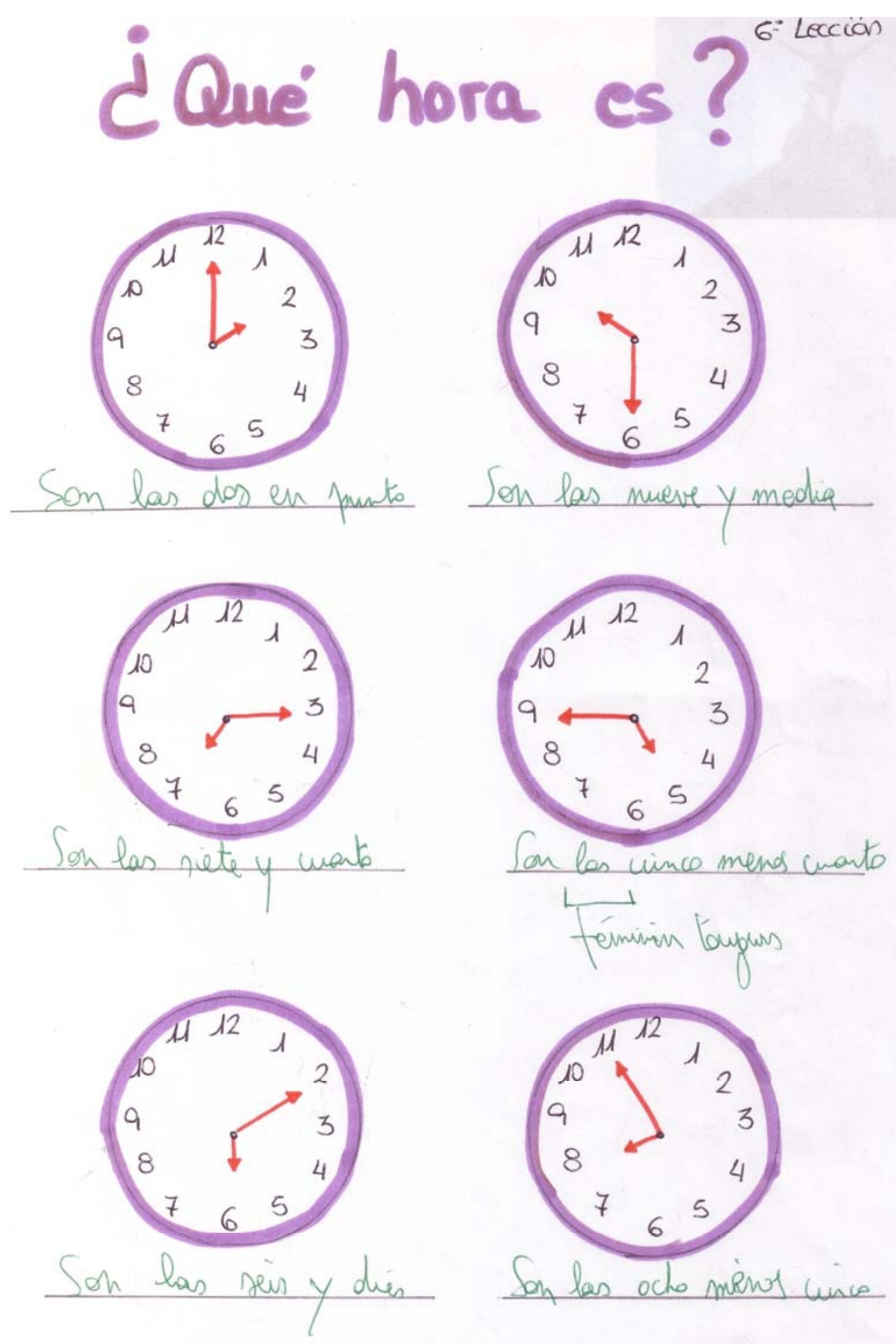

\section{Ilustración 3 (Actividad para trabajar las horas del reloj)}

\section{Fomentar las relaciones interpersonales y sociabilidad (comunicación)}

Desde el año 1939 (Lewin) se cita la dinámica de grupo. Un grupo se compone de varias personas, entre las que existe una interacción y se rige por unas normas y objetivos a alcanzar. Shaw (1971) definía el grupo como "Dos o más personas que interactúan entre ellas, en forma tal que cada uno influye en y es influido por la otra persona” ${ }^{13}$.

\footnotetext{
${ }^{13}$ Jesús Gómez Tolón y Roberto Salvanés Pérez. Terapia ocupacional en psiquiatría. Zaragoza, Mira editores, 2003. Pág 31-32
} 
Los pacientes afectados de trastornos psiquiátricos en muchos casos presentan problemas en el mantenimiento de relaciones estables, tanto con el Terapeuta Ocupacional como con el resto de compañeros. Esto, junto a la inestabilidad emocional que presentan, interfiere en la realización de actividades grupales.

En los pacientes con los que trabajábamos este aspecto estaba muy deteriorado, lo que podía observarse en que la mayor parte de ellos optaba por actividades individuales para llevar a cabo en Terapia Ocupacional. Por ello nos pareció muy interesante potenciar este aspecto planteando actividades grupales que implican interacciones y en las que se establecen relaciones de ayudaapoyo y dependencia-liderazgo. Así se conseguía la educación en la percepción social por medio de empatía y sensibilidad hacia los otros.

Por otra parte intentamos mejorar las aptitudes de comunicación procurando hacer declaraciones claras entre ellos; hablando solo una persona en cada momento; que todos los miembros del grupo formen parte de la conversación de forma equivalente; dirigirse a los compañeros directamente, no hablando en tercera persona.

El objetivo principal fue que los pacientes aprendieran a través de la resolución de los problemas grupales a colocarse en la posición del otro, provocando la necesidad de que consideraran las múltiples causas de la conducta de los demás, las consecuencias de dichas conductas y los diversos métodos para resolver, por su parte y por parte de los demás, el problema ocupacional que se le plantea al grupo de trabajo.

\section{Mejorar el autoconcepto y la autoestima}

La ansiedad que estas personas experimentan al realizar ciertas actividades conducen a no iniciar las mismas, llegando a pedir ayuda a otras personas para realizarlas. Este hecho facilita que se produzca un proceso de pérdida de la autoestima percibiéndose a sí mismo como incompetente. En ellos el autoconcepto se encuentra desestructurado, que se observa en la alteración de los distintos tipos del "self” (erótico, somático, actitudinal y aptitudinal) en el ámbito ocupacional.

La actividad con objetivos previamente seleccionados va a determinar, gracias a su valor comunicacional con los demás y con uno mismo, una modificación sobre el autoconcepto. La actividad ocupacional, va a modificar el concepto que los demás tienen sobre el sujeto de la acción y esta "opinión” ajena va a repercutir sobre el autoconcepto de la persona actuante. La aceptación o no de esta opinión ajena, y los mecanismos de protección y defensa que pueda generar el sujeto ante la misma, van a dar un resultado de equilibrio mental en permanente cuestionamiento ${ }^{14}$.

Para mejorar su autoconcepto y autoestima tratamos de convertir las clases de español en una actividad positiva hacia la propia persona, en la que ella misma experimentaba una sensación de seguridad, identidad, pertenencia y significado. El uso de refuerzos positivos por parte del terapeuta o los componentes del grupo, tanto verbales ("muy bien”, “ánimo”, "sigue así”, "aplauso") como materiales (diplomas, fiesta final) y las críticas siempre constructivas fueron algunos de los instrumentos utilizados para conseguir que el paciente experimentara éxito en las sesiones. Con ello aumentaba su autoestima viéndose motivado para volver a la clase siguiente.

${ }^{14}$ Jesús Gómez Tolón .Fundamentos metodológicos de la terapia ocupacional. Zaragoza, Mira editores, 1997 Pág 121 
Dear

You are invited to the last spanish activities during next Friday, $5^{\text {th }}$ of December with Patricia and Cristina.

In the morning (10.30-11.30): we have prepared a remember's Game using all spanish words that you know.

Come and join us, it will be funny!!!!

In the afternoon (13.00-15.00): we will learn to coock a tipycal spanish meal:

tortilla de patata,

and then we have a snack while we listen

to spanish music.

We are looking forward to see tou there!!

\section{Patricia \& Cristina}

\section{Ilustración 4( Invitacion personal a la fiesta española)}

\section{Potenciar la función ocupacional}

Siguiendo el Modelo de Ocupación Humana de Gary Kielhofner, los individuos son activos en términos de ocupación cuando satisfacen sus propias necesidades de exploración y dominio y cumplen las necesidades sociales de participación productiva y de ocio. La disfunción ocupacional se produce en el enfrentamiento del individuo con el entorno, por una disminución, ineficacia o desequilibrio de la conducta ocupacional ${ }^{15}$. Según el MoHO la disfunción ocupacional se manifiesta en factores que pueden incluir lesiones en:

\footnotetext{
${ }^{15}$ Gary Kielhofner. A Model of Human Occupation: Theory and application $2^{\text {nd }}$ ed. Maryland., Williams \& Wilkins, 1995.
} 
-Alteración de la Volición

-Pérdida de hábitos y roles

-Subsistema de ejecución mente-cerebro-cuerpo

-Dificultades en las relaciones de las personas con el ambiente

Así pues nos propusimos conseguir unos objetivos específicos, cada uno de los cuales hace referencia a uno de los subsistemas propuestos por G. Kielhofner:

\section{Aumentar la motivación}

Uno de los síntomas típicos de estas personas es la existencia de un estado hipomotivacional. Partiendo de esta base, plantear actividades que les motiven resulta complicado. En nuestro caso el hecho de que fuéramos estudiantes Erasmus, que viniéramos de España y lo novedoso de nuestra presencia allí propició una positiva motivación inicial.

La actividad en sí misma es una de las claves para conseguir la motivación. Actividad que debe ser propositiva y significativa para las personas que la llevan a cabo. Como ya se conocía previamente, el aprendizaje de español cumplía estas características.

Otra de las claves para aumentar la motivación es la actitud del Terapeuta Ocupacional para con los pacientes. Nos dimos cuenta de que con este tipo de personas el que vinieran o no a las clases dependía en gran parte de nosotras. En un principio teníamos que ser capaces de motivarles para que vinieran a la clase, para lo cual una de las actitudes tomadas era ir a buscarles todos los días e invitarles a venir. Posteriormente, había que mantener esta motivación dentro de cada sesión haciéndolas divertidas e interesantes. El uso de diplomas como refuerzo positivo que se darían al final del curso fue utilizado para potenciar la motivación a largo plazo, motivación que debe ser mantenida.

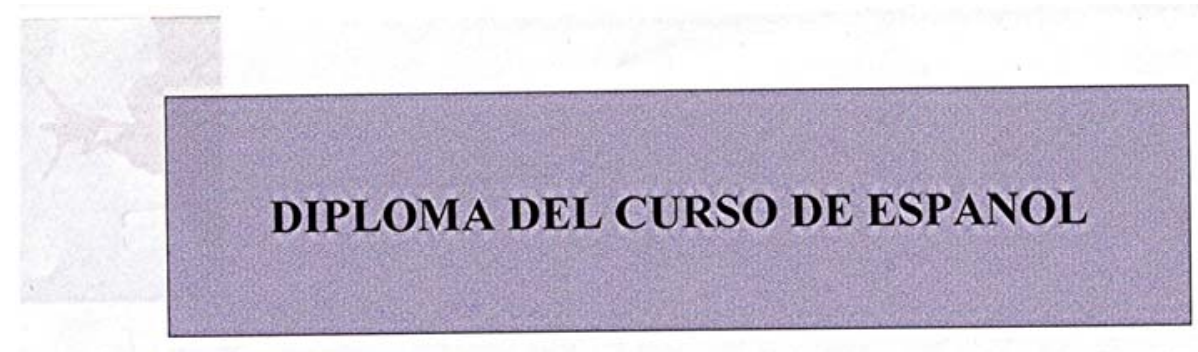

- Dhr/mevr......................................

- volgde de cursus Spaanse taal voor beginners met groot enthousiasme van 3 oktober tot 5 december 2003.

Teachers: Patricia Jovellar / Cristina de Diego

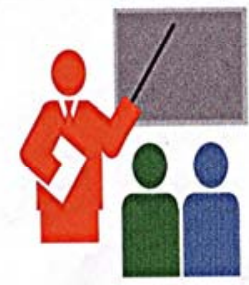

\section{Ilustración 5 (Diploma de realización del curso)}

Este mantenimiento es uno de los aspectos más difíciles de conseguir pero es también un pilar básico si se quiere obtener verdaderos resultados terapéuticos. Dar continuidad a las actividades es la única manera de lograrlo. Por ello decidimos, tras finalizar las prácticas en Kortenberg, continuar con las clases de español pero esta vez a distancia (un nuevo reto para nuestros alumnos. 


\section{Instaurar hábitos y roles}

Este subsistema se ve muy alterado. Existe una gran dificultad en la identificación de los propios roles a pesar de que muchas veces los tengan, así como en la instauración de hábitos por todo lo que ello conlleva.

Reforzar y apoyar este subsistema nos pareció muy importante ya que los hábitos y los roles organizan, regulan y mantienen el comportamiento para ayudar a la persona a satisfacer los estándares sociales y le permiten adaptarse al medio ${ }^{16}$.

Desde las clases de español se podían acostumbrar a reforzar el habito que implica el ir a Terapia Ocupacional dos días fijos a la semana de forma regular y a unas horas determinadas. Al estar una hora haciendo esta actividad de forma continuada, se fomentaba la interiorización del rol de estudiante. En todo momento se trató de reentrenarles en una serie de habilidades y destrezas que les permitieran ser capaces de empezar nuevas actividades, en un principio dentro de la unidad de Terapia Ocupacional y después fuera de la clínica si era posible.

\section{Fomentar la toma de decisiones y responsabilidad}

La toma de decisiones, se ve muy alterada en estas personas, lo cual repercute en la dificultad para iniciar acciones e intentar cosas nuevas. Si la decisión de iniciar la acción finalmente se llevara a cabo, es frecuente el incumplimiento de lo pactado, lo que pone de manifiesto la no ejecución de la misma. En general, cuando llevan a cabo las tareas no valoran los resultados, por lo que se les hace difícil corregir sus errores ${ }^{17}$.

Nos pareció muy significativo el hecho de que personas con estas características, tras la propuesta de iniciar las clases de Español como actividad, fueran capaces de tomar por ellos mismos la decisión de venir, y, algunos de ellos, asumir posteriormente la responsabilidad para hacerlo de forma continuada.

Para valorar este aspecto se utilizó un registro de asistencia en el que se indicaba con una "V" la asistencia completa y activa a cada sesión y con una " $\mathrm{X}$ " si esta era incompleta o con interrupciones.

\footnotetext{
${ }^{16}$ Hopkins,H.; Smith, H.: Terapia ocupacional. Madrid, Editoria1 Médica Panamericana, 1998.

${ }^{17}$ Jesús Gómez Tolon y Roberto Salvanés Pérez. Terapia ocupacional en psiquiatría. Zaragoza, Mira editores, 2003.
} 


\section{SPANISH LESSONS}

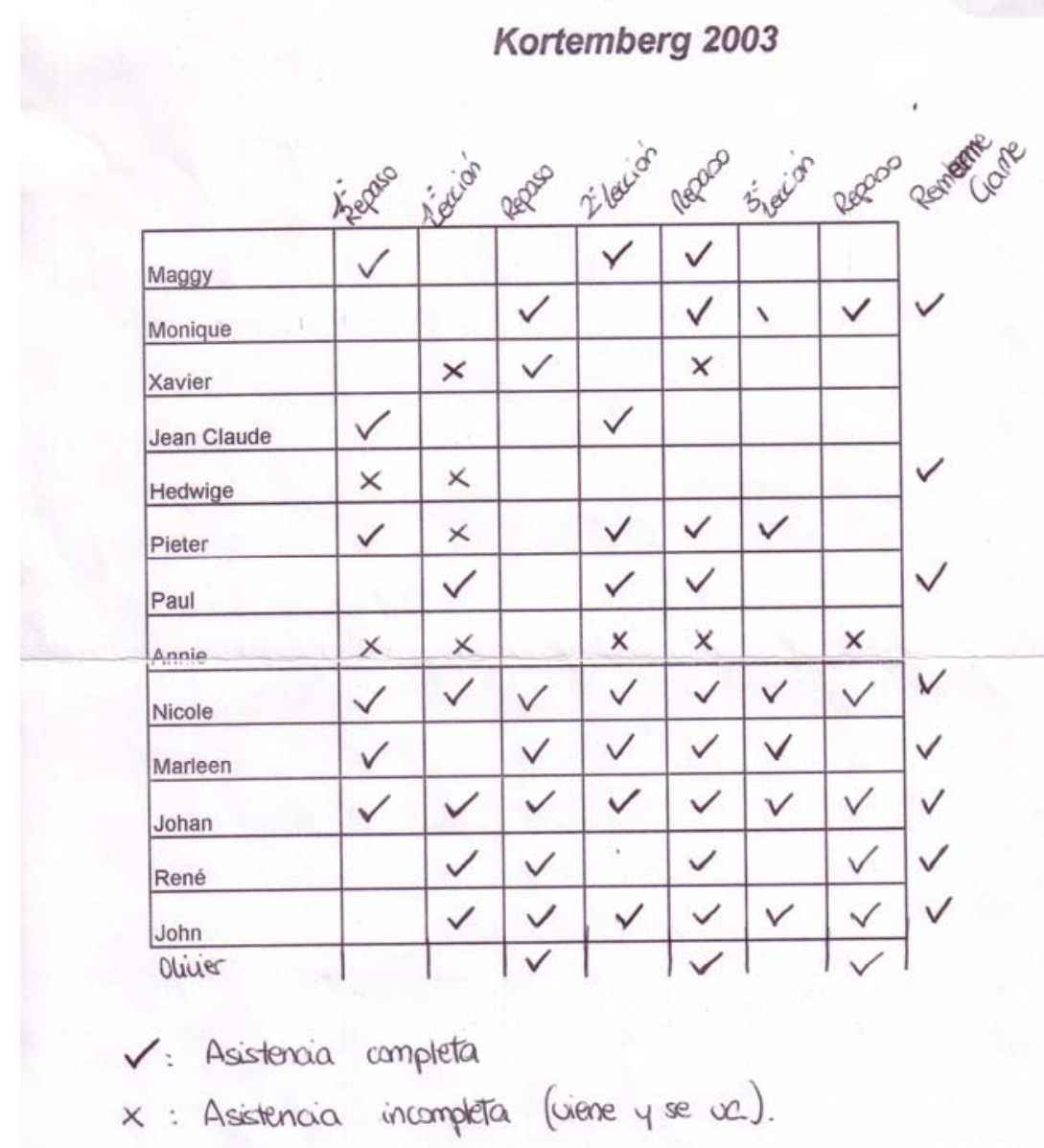

Pagina 1

\section{Ilustración 6 (Control de asistencia)}

\section{Aminorar la incidencia de las alucinaciones auditivas}

Muchos de los pacientes con los que trabajábamos en las clases padecían esquizofrenia. Estas personas manifiestan frecuentemente los síntomas positivos de la enfermedad, así llamados porque añaden algo a la experiencia que se tiene del mundo.

Nos referimos concretamente a las alucinaciones, que pueden ocurrir en cualquier modalidad sensorial (auditiva, visual, olfativa, gustativa y táctil), pero las alucinaciones auditivas son, con mucho, las más habituales y características. Son experimentadas generalmente como voces, ya sean conocidas o desconocidas, que son percibidas como distintas de los pensamientos de la propia 
persona. El contenido puede ser bastante variable, aunque son especialmente frecuentes las voces peyorativas o amenazantes ${ }^{18}$.

Slade y Bentall argumentan que en el tratamiento de alucinaciones desde una aproximación psicológica se puede hacer uso de la Terapia Distractoria. Esta terapia se basa en que, hipotéticamente, cuando la persona es capaz de distraerse de sus voces, estas deberían tender a la extinción ${ }^{19}$. La distracción requiere que la persona ignore las voces y se concentre en actividades que requieran cierto nivel de atención, como pueda ser leer, escuchar la radio o juegos aritméticos y mentales. Estas actividades dificultan que el paciente preste atención al contenido y significado de las voces. Evidencias de estudios anteriores indican que esta técnica le da a la persona mayor control sobre sus voces y sería efectiva en la reducción de la severidad de la persistencia de las alucinaciones auditivas. Aunque nunca se conseguiría su eliminación total.

Anteriormente hemos expuesto la estructura de las clases y algunas de las actividades que en ellas se llevaban a cabo, quedando de manifiesto que estas actividades cumplen las características necesarias como para formar parte de una Terapia Distractoria. Nuestra intención era dar a los pacientes la oportunidad de convertir las clases de español en su propia "estrategia de copia”, con la cual pudieran aprender a controlar sus voces y vivir de la mejor manera posible con los síntomas residuales de su enfermedad.

\section{Caso práctico: a propósito de J.L}

J.L. es un varón de 45 años de edad que padece esquizofrenia desde el año 1990. En 1997 fue admitido por primera vez en Terapia Ocupacional de la unidad de Saint Franciscus. La única actividad que realizaba en la unidad desde hacía largo tiempo era jugar al solitario en el ordenador. J.L. realizaba esta actividad de forma rutinaria e individual. Su comunicación con los demás era muy escasa, sin tener iniciativa para entablar conversaciones y, si lo hacía, era sólo a base de monosílabos. Así que las relaciones con sus compañeros y con el terapeuta eran casi nulas. Al entrar en la sala de Terapia Ocupacional evitaba el contacto visual y casi nunca saludaba ni se despedía, acostumbraba a andar con la cabeza agachada y cara inexpresiva.

A pesar de las propuestas del terapeuta para cambiar de actividad, él nunca las aceptaba. Cual fue la sorpresa del Terapeuta Ocupacional cuando con nuestra llegada su actitud empezó a cambiar. Días antes de comenzar las clases motivamos e invitamos a todos los pacientes a participar en ellas. En el caso de J.L. la respuesta siempre era la misma: “ya lo pensaré”. Fue muy grato el descubrir que muchas personas habían tomado la iniciativa de venir el primer día de clase, pero J.L. no estaba allí. Diez minutos bastaron para que encontrara la clase.

El objetivo que nos planteamos conseguir con él fue la consecución de un comportamiento progresivo hacia un mayor grado de participación y asertividad, vigilando en todo momento la aparición de aislamiento o rechazo por parte del resto del grupo, expresivos de una descalificación o no aceptación.

Para alcanzar este objetivo fue decisiva la relación terapéutica que establecimos con J.L. basada en un compromiso dinámico, adaptable a cada momento del tratamiento, para así solucionar los posibles problemas que pudieran surgir. Cabe desatacar la importancia de su participación activa para alcanzar la meta terapéutica deseada.

Con este nuevo hábito logramos que J.L. recuperara un elemento importante en su vida que años atrás había perdido: la adquisición de nuevos roles, en este caso el de estudiante. J.L. fue experimentando éxito en las clases, lo que repercutía positivamente en su autoestima y autoconcepto. Esto le hizo ser consciente de que podía comenzar actividades nuevas y asumir distintas responsabilidades.

Fue muy significativo el comportamiento de J.L. durante las actividades que realizamos el día de “fin de curso". Por la mañana se planteó un “Remember's game” de todo lo aprendido.

\footnotetext{
${ }^{18}$ DSM-IV: Manual diagnostico y estadístico de los trastornos mentales $4{ }^{a}$ ed. Barcelona, Masson S.A, 1995. Pág 281

${ }^{19}$ Haddock G, Slade PD, Bentall RP, Reid D, Faragher EB. A comparision of the long term effectiveness of distraction and focusing in the treatment of auditory hallucinations. British Journal of Medical Psychology 1998, September 71 (Pt3): 339-349.
} 


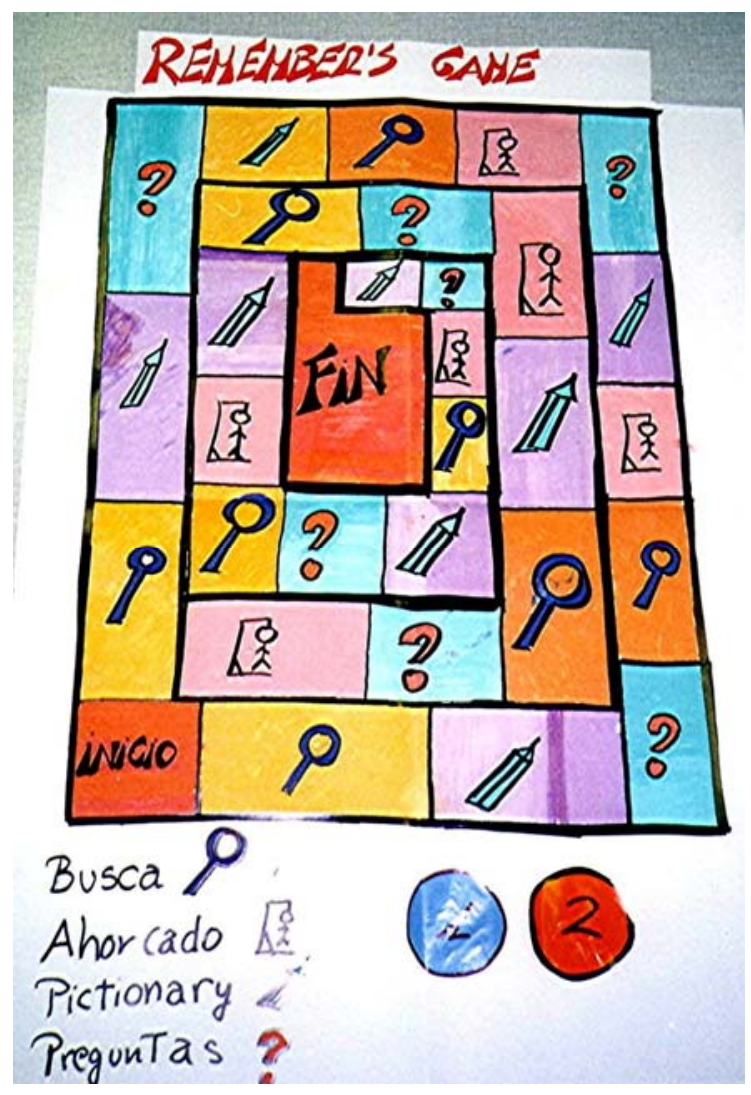

Ilustración 7 (Remember's Game)

Son muchos los profesionales que han reconocido el potencial terapéutico del juego: desde Terapia Ocupacional se considera al juego como un medio natural de desarrollo y autoexpresión que da la oportunidad de exteriorizar los sentimientos de tensión, frustración, inseguridad, agresión, temor, perplejidad y confusión. Con el juego se adquieren experiencias de las cosas que nos rodean, del medio donde se juega, se desarrollan destrezas de coordinación y subordinación al grupo en el que se está y el sentido de cooperación con los demás.

Se ha analizado el juego desde diversos puntos de vista, nombramos a continuación los más relevantes teniendo en cuenta nuestra aproximación terapéutica:

- $\quad$ Catártico: va encaminado a purificar, limpiar tendencias nocivas que es necesario descargar. Se necesita de alguna manera dar salida a la agresividad acumulada. Este tipo de juego se encarga de canalizar o derivar estas tendencias nocivas dándoles una salida inofensiva ${ }^{20}$.

- $\quad$ Preparatorio: el juego en sí mismo es un ejercicio preparatorio para la vida adulta que con frecuencia se ve deteriorada en estas personas.

- $\quad$ Actividad social: a través del juego se reconstruyen las relaciones sociales.

Para llevar a cabo el "Remember's Game" se hicieron dos equipos que alternativamente iban solucionando pruebas (adivinanzas, ahorcado, reconocimiento visual de un objeto dibujado o discriminación de un objeto trabajando las habilidades espaciales dentro de la sala). Dicho juego fue una manera de valorar la capacidad de los pacientes para poner en práctica todos los aspectos trabajados durante las clases.

Por la tarde se hizo una "Fiesta Española". Entre todos cocinamos tortillas de patata, apoyándonos en una receta debidamente estructurada y trabajada previamente. Fue sorprendente la

\footnotetext{
${ }^{20}$ Romero Ayuso, D.; Moruno Miralleres, P. Terapia ocupacional: Teoría y Técnicas. Barcelona, Masson, 2003. Pág. 401-402
} 
participación, colaboración e interacción que todos ellos establecieron este último día. Ellos mismos fueron capaces de organizarse distribuyéndose las tareas para conseguir una finalidad común: pasar un rato agradable y divertido aprendiendo un poco de cultura española.

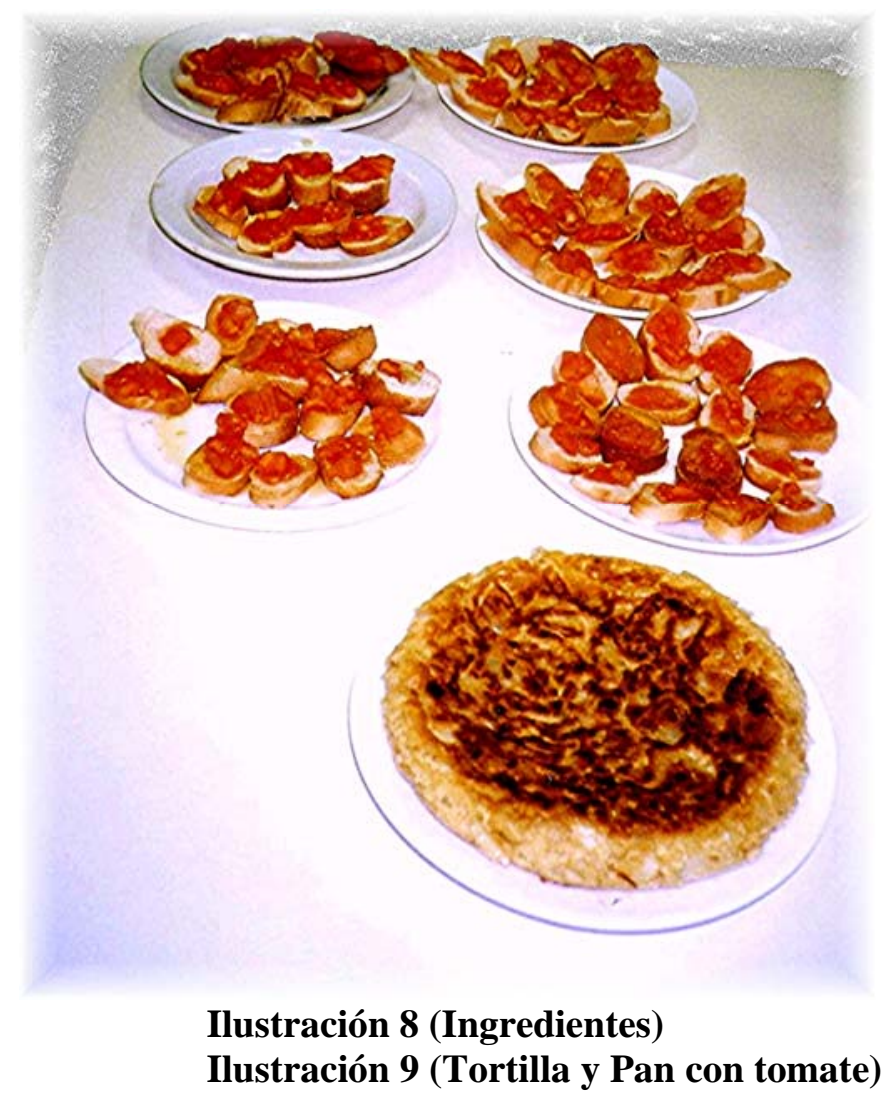

Poco a poco J.L. se convirtió en uno de las personas más participativas y constantes, mostrando en los días sucesivos un gran interés. Sus relaciones interpersonales iban mejorando, incluso llegó a establecer relaciones de ayuda-apoyo con sus compañeros. También pudimos ver un claro progreso en su comunicación y expresividad: se le oía reír en clase y ya nunca olvidaba saludarte o despedirse cuando acudía a Terapia Ocupacional. La postura nos reveló un cambio en su actitud: de llevar los hombro caídos con el pecho deshinchado (signo de estado de ánimo bajo) pasó a adoptar una postura más erguida con los hombro hacia atrás. Finalmente J.L. empezó a interiorizar herramientas de comunicación no verbal como la expresión facial y la mirada para reforzar su interacción verbal con los demás.

Tras nuestra marcha, J.L. siguió acudiendo a Terapia de forma regular para continuar su aprendizaje de español mediante las clases a distancia y con programas de ordenador destinados a ello.

Ahora ya han pasado dos años desde que la actitud de J.L. empezó a cambiar, actualmente vive en una casa protegida y acude diariamente al centro "St Maarten" donde realiza diversas actividades.

Es sorprendente cómo en ocasiones una pequeña iniciativa y muchas ganas de trabajar pueden cambiar el transcurso de toda una vida. Así fue la historia de cómo comenzar otra vez y encontrar un nuevo equilibrio ocupacional y bienestar en la vida.

\section{Conclusiones}

Con las clases de español como actividad terapéutica pretendimos proporcionar a estas personas las herramientas necesarias para que aprendieran cómo obtener experiencias positivas y satisfactorias de la ocupación. Cuando a través de la ocupación llegas a la 
autosatisfacción personal, esto repercute positivamente en tu calidad de vida. Es entonces cuando la ocupación significa mucho más: una constante toma de decisiones, el planteamiento de una serie de metas u objetivos a cumplir a largo y corto plazo, una motivación, interés e incentivación en tu vida que hace que te sientas útil y que te identifiques con un roles ayudándote a estructurar la vida mediante hábitos y rutinas.

Es muy importante mencionar que el aprendizaje de un idioma proporciona de forma natural la adquisición de una amplia gama de vocabulario, favoreciendo de manera espontánea la comunicación.

Este proyecto supuso una experiencia muy positiva, tanto para nosotras, desde el punto de vista de nuestra formación, como para ellos, en cuanto a la consecución progresiva de los objetivos planteados. En vista de lo cual decidimos, junto con el Terapeuta Ocupacional, continuar con esta iniciativa mediante el “Curso de Español a Distancia”, un escalón más en su periodo de rehabilitación. Cada semana trabajaban solos con los ejercicios que les mandábamos desde España apoyándose en un diccionario y un programa de ordenador. El terapeuta era el encargado de remitirnos cada lección. Nosotras las corregíamos y se las volvíamos a mandar con anotaciones para motivarles a continuar con su trabajo.

Tras el éxito obtenido con este "Curso a Distancia" las clases fueron retomadas por los sucesivos estudiantes Erasmus que iban llegando al hospital de Kortenberg. Incluso ahora, dos años más tarde, se continua con las clases de español en varias unidades. Los objetivos han cambiado en función del tipo de pacientes (psicóticos y abusos) y sus necesidades, lo que hace que ahora estén más dirigidos al entrenamiento cognitivo.

El hecho de dar esta continuidad a las clases de español supone una de las características más importantes de lo que consideramos actividad terapéutica. De este modo el proceso de rehabilitación tiene como finalidad la progresión en el alcance de metas cada vez mayores y más próximas a lo que supone una verdadera vida en sociedad. Ahora los pacientes tienen las herramientas necesarias para poder iniciar una actividad de similares características, pero esta vez, fuera del hospital.

Como ultima reflexión nos gustaría animar a todos los Terapeutas Ocupacionales a compartir sus conocimientos y experiencias con otras culturas en las que se desarrolle la Terapia Ocupacional, con la finalidad de que todos juntos trabajemos en la misma línea de actuación y con los mismos objetivos. Esta es la única manera de conseguir una práctica terapéutica centrada en lo verdaderamente importante: la persona.

En nuestra situación de estudiantes, el Erasmus nos dio la oportunidad de conocer la práctica de la Terapia Ocupacional en Bélgica, suponiendo una experiencia inolvidable.

\section{Agradecimientos}

Nos gustaría dar las gracias al Terapeuta Ocupacional Luc Vercruysse por su apoyo incondicional y por todo lo que nos enseñó durante nuestra estancia en Bélgica.A Clara Sánz, Terapeuta Ocupacional de la Universidad de Zaragoza, por darnos un punto de vista más actual y práctico de la Terapia Ocupacional.

\section{Bibliografía}

- Kielhofner G. Model of Human Occupation: Theory and application . 3rd ed. Lippincott, Williams \&Wilkins, 2002.

- DSM-IV: Manual diagnostico y estadístico de los trastornos mentales. $4^{\mathrm{a}}$ ed. Barcelona, Masson S.A, 1995

- Gómez Tolon J, Salvanés Pérez R. Terapia ocupacional en psiquiatría. Zaragoza, Mira editores, 2003.

- Gómez Tolón J .Fundamentos metodológicos de la terapia ocupacional. Zaragoza, Mira editores, 1997. 
- Gómez Tolón J. Habilidades y destrezas en terapia ocupacional. Zaragoza, Mira editores, 2000.

- Kielhofner G. A Model of Human Occupation: Theory and application. $2^{\text {nd }}$ ed. Maryland, Williams \& Wilkins, 1995.

- Hopkins H, Smith H. Terapia ocupacional. Madrid, Editorial Médica Paramericana, 1998.

- Haddock G, Slade PD, Bentall RP, Reid D, Faragher EB. A comparision of the long term effectiveness of distraction and focusing in the treatment of auditory hallucinations. British Journal of Medical Psychology 1998, September 71 (Pt3)

- Romero Ayuso D, Moruno Miralleres P. Terapia ocupacional: Teoría y Técnicas. Barcelona, Masson, 2003.

- British Journal of Medical Psychology 1998, September 71

- de Hert M, Magiels G, Thys E. The secret of the brain chip. A self-help guide for people experiencing psychosis. Antwerpen, EPO, 2003.

- Punwar Alice J. Peloquin Suzanne M. Occupational Therapy: principles and practice. Lippincott, 2000. 